\title{
A model for underpotential deposition in the presence of anions
}

\author{
M. C. Giménez, ${ }^{1, a)}$ A. J. Ramirez-Pastor, ${ }^{1}$ and E. P. M. Leiva ${ }^{2}$ \\ ${ }^{1}$ Departamento de Física, INFAP, Facultad de Ciencias Físico-Matemáticas y Naturales, \\ Universidad Nacional de San Luis, CONICET, Chacabuco 917, 5700 San Luis, Argentina \\ ${ }^{2}$ Departamento de Matemática y Física, Facultad de Ciencias Químicas, Universidad Nacional de Córdoba, \\ 5000 Córdoba, Argentina
}

(Received 17 February 2010; accepted 19 April 2010; published online 13 May 2010)

\begin{abstract}
A simple model to study the effect of on top coadsorption of anions in underpotential deposition is formulated. It considers a lattice-gas model with pair potential interactions between nearest neighbors. As test system, the electrodeposition of silver on gold is studied by means of grand canonical Monte Carlo simulations. The influence of anions on the adsorption isotherms is analyzed. It is found that as the interaction between silver atoms and anions increases, the monolayer adsorbs at more negative chemical potentials. For large interactions between silver atoms and anions, a expanded structure occurs for the silver monolayer. () 2010 American Institute of Physics.

[doi:10.1063/1.3427585]
\end{abstract}

\section{INTRODUCTION}

The study of the adsorption of particles on surfaces is important from the point of view of surface science and due to its potential applications in nanotechnology and catalysis. From the electrochemical point of view, it is of great interest to study the electrodeposition of a metal onto a single crystalline surface of a foreign metal. ${ }^{1-4}$ When this occurs at potentials more positive than those predicted from the Nernst equation, the process is denominated underpotential deposition (UPD). ${ }^{1,3,5-7}$ In the case of voltammetric profiles, UPD processes are evident by the occurrence of one or more current peaks, which can be related to the existence of different structures (phases) covering the surface of the substrate.

One of the open questions in electrochemistry is the fact that theoretical models and computer simulations predict first order phase transitions for the electrodeposition of one metal on the surface of another metal. ${ }^{8,9}$ Nevertheless, in the experimental voltamograms, the observed peaks are generally wide and not sharp. Another experimental observation that is not well explained by theory is the existence of different commensurate structures for the same adsorbate/substrate couple, like those occurring in the case of Ag adsorption on $\mathrm{Au}(111)$ studies. In this particular system, in situ techniques such as atomic force microscopy, ${ }^{10}$ scanning tunneling microscopy, ${ }^{11-13}$ and others (see review ${ }^{14}$ ) have allowed the study of a wide range of structures. All these studies report the existence of a compact $\mathrm{p}(1 \times 1)$ structure at potentials very close to the bulk silver deposition and a series of more open structures at higher potentials up to $0.60 \mathrm{~V}$ more positive than the bulk deposition. Similar findings were made in the case of the deposition of $\mathrm{Ag}$ on $\mathrm{Au}(100),{ }^{12}$ where also relatively open structures occur at high underpotentials. It must be pointed out that the existence of two-dimensional

\footnotetext{
${ }^{\text {a) }}$ Author to whom correspondence should be addressed. Present address: IFEG, CONICET, FaMAF, Universidad Nacional de Córdoba, C.P. 5000 Córdoba, Argentina. Tel.: 0054-351-4334051. Electronic mail: ceciliagim@gmail.com.
}

structures with a lattice parameter larger than that of the substrate is rather unexpected in the case of $\mathrm{Ag} / \mathrm{Au}(\mathrm{hkl})$ systems since the lattice misfit of the atoms involved is practically negligible. These superlattice structures, commensurate with the substrate but with overlayerstructures larger than the $(1 \times 1)$ structure, are usually denominated expanded structures. ${ }^{3}$ First principles calculations have shown that none of the expanded structures found in $\mathrm{Au}(111)$ should be thermodynamically more stable than the $\operatorname{Ag}(1 \times 1) / \mathrm{Au}(111)$ phase, ${ }^{15}$ a fact that is challenged by their electrochemical stability. To explain this contradiction, it has been proposed in Ref. 15 that anion coadsorption, promoted by the shift of the potential of zero charge upon adatom formation, could be aiding the occurrence of the expanded UPD layers. A similar explanation has been proposed before by Sanchez and Leiva ${ }^{16}$ to explain the occurrence of UPD in the case of the $\mathrm{Cu} / \mathrm{Au}(111)$, which is a system where the binding energy of $\mathrm{Cu}$ on $\mathrm{Au}(111)$ is considerably lower than the bulk binding energy of $\mathrm{Cu}$. Concerning the case of the system $\mathrm{Cu} / \mathrm{Au}(111)$ in sulfuric acid solutions, system-specific models have been developed that successfully describe the voltammetric behavior by means of a lattice model simulation ${ }^{17}$ or the application of statistical mechanics tools. ${ }^{18}$ In both cases, the interaction parameters involved were fitted for this specific system.

In the present work, rather than attempting to simulate an UPD system in the presence of a specific anion type, we will use metal-anion and anion-anion interactions as parameters. We use this approach in order to understand some essential features of the physics involved in the coadsorption processes. In this regard, the aim of the present model is not to present a detailed description of reality, but rather to show that taking into account only some essential features of the system, some experimental facts can be explained. More important, the magnitudes of the parameters used in the work describing metal-metal interactions correspond to values that are physically sound and not artificially fitted to a given system. 
Concerning the atoms of the adsorbate and the substrate, we consider interaction parameters that are typical for the $\mathrm{Ag} / \mathrm{Au}(100)$ system. It is important to emphasize that these parameters were not obtained to fit the properties of the electrochemical system, but were derived from calculations performed with realistic (many body) interatomic potentials.

The outline of the paper is as follows. In Sec. II we describe the lattice-gas model along with the simulation scheme. In Sec. III we present the results. Finally, the general conclusions are given in Sec. IV.

\section{MODEL AND SIMULATION METHOD}

\section{A. Lattice model}

Lattice models for computer simulations are very useful in studies of adsorption on surfaces because they allow dealing with a large number of particles at a relatively low computational cost. ${ }^{19,20}$ For this reason, we will use this approach to tackle the study of the present problem.

The system $\mathrm{Ag} / \mathrm{Au}(100)$ has been largely studied, both experimentally $^{12,13,21,22}$ and theoretically. ${ }^{8,23,24}$ As stated above, it is well known that the crystallographic misfit between the involved atoms is not important for this system. Thus, it is a good approximation to assume that the adatoms adsorb on defined discrete sites on the surface, given by the positions of the substrate atoms. Two epitaxial square lattices with periodical boundary conditions are used in the present approach. The first monolayer can be occupied by silver atoms and it is in contact with the gold surface. The second one can be occupied by anions and they can interact among them and with the silver atoms that are localized in the first monolayer.

According to this picture, the interaction of the anions with the bare substrate is neglected. Experimental results show in fact that in many cases anion adsorption on the substrate is small or negligible in the potential range where the UPD layer is formed. For example, Fig. 3(b) of the manuscript by Shi et $a .^{25}$ shows that a $0 \mathrm{mV}$ versus saturated calomel electrode (SCE) sulfate and chloride adsorption on $\mathrm{Au}(111)$ are negligible, and moderate for $\mathrm{Br}^{-}$. The same figure shows that anion adsorption (of all types) is strongly enhanced by the presence of adatoms, a fact that is born by the physics in our model.

The simulations are performed in the grand canonical ensemble for the two components. This means that the number of adsorbate atoms as well as the number or anions can fluctuate. Each lattice point in the first monolayer represents an adsorption site for an adsorbate atom and each lattice point in the second monolayer represents a site for anion adsorption just on top of the site of an adsorbate atom.

\section{B. Energy calculations}

The energy $E$ of the system is calculated within the classical lattice-gas model with pair potential interactions between nearest neighbors, but, in the present case, we take into account two epitaxially superimposed lattices.

If we denote metal atoms as type 1 species and anions as type 2 species, the different interactions involved are the following.
- Adsorbate-surface (attractive) interactions: $E_{\text {ads, }}$,

- adsorbate-adsorbate (attractive) interactions: $J_{11}$,

- adsorbate-anion (attractive) interactions: $J_{12}$, and

- anion-anion (repulsive) interactions: $J_{22}$.

Based on our previous work, ${ }^{26}$ where we approximated many body potentials by means of effective pairwise interactions, we have considered here $E_{\text {ads }}=-2.58 \mathrm{eV}$ and $J_{11}=-0.28 \mathrm{eV}$, which are parameters suited to describe the $\mathrm{Ag} / \mathrm{Au}(100)$ system.

The values of $J_{12}$ and $J_{22}$ were taken as parameters that can be varied. In order to estimate the order of magnitude for $J_{12}$, we can consider the Coulombic attraction between two point charges,

$$
J_{12} \approx \frac{1}{4 \pi \epsilon_{0}} \frac{Q_{1} Q_{2}}{r} .
$$

As a first estimation, we can take $Q_{1} \approx 1$ (for the $\mathrm{Ag}^{+}$ cation), $Q_{2} \approx-1$ (for the anion) and $r \approx 1 \mathrm{~nm}$. This gives an interaction energy of $J_{12} \approx-1.4 \mathrm{eV}$. On the other hand, if we consider $Q_{1} \approx 0.1$ (for the $\mathrm{Ag}$ cation, taking into account a partial charge transfer) and $r \approx 0.3 \AA$ (for a more realistic distance), we obtain $J_{12} \approx-0.5 \mathrm{eV}$.

This simple analysis points to give an order of magnitude expected for this constant. However, both constants $\left(J_{12}\right.$ and $\left.J_{22}\right)$ are taken as parameters that can take any value and this paper explores the implications of different possible values.

If we label with $\mathrm{cm}_{\alpha}$ the occupation state of site $\alpha$ in the first monolayer and with $c a_{\alpha}$ the occupation state of site $\alpha$ in the second monolayer (just above the $\alpha$ site in the first monolayer), we can write the Hamiltonian as follows:

$$
\begin{aligned}
E= & \sum_{\alpha} E_{\mathrm{ads}} \delta_{c m_{\alpha}, 1}+\sum_{\langle\alpha, \beta\rangle} J_{11} \delta_{c m_{\alpha}, 1} \delta_{c m_{\beta}, 1} \\
& +\sum_{\alpha} J_{12} \delta_{c m_{\alpha}, 1} \delta_{c a_{\alpha}, 2}+\sum_{\langle\alpha, \beta\rangle} J_{22} \delta_{c a_{\alpha}, 2} \delta_{c a_{\beta}, 2},
\end{aligned}
$$

where the symbol $\delta$ represents the Kronecker delta and the notation $\langle\alpha, \beta\rangle$ denotes a sum taking account of all pairs of nearest neighbors.

This Hamiltonian is proposed on the basis of experimental evidence found by Shi et al. ${ }^{27}$ Figure 3 of this manuscript shows that at $0 \mathrm{mV}$ versus SCE sulfate and chloride adsorption on $\mathrm{Au}(111)$ are negligible, and moderate for $\mathrm{Br}^{-}$. The same figure shows that anion adsorption (of all types) is strongly enhanced by the presence of adatoms, a fact that is born by the physics in our model. The present model could be straightforwardly generalized to consider anion adsorption on the substrate using a three-state model (Ag, anion, or empty) for layer 1, coupled to a two-state model (anion or empty) for layer 2 .

\section{Grand canonical Monte Carlo simulation}

Two superimposed square lattices of $M=L \times L$ adsorption sites with periodical boundary conditions are used here to represent the first monolayer, just above the gold surface 
(to be occupied by silver atoms) and the second monolayer, just above the silver monolayer (to be occupied by anions).

The simulations are performed in the grand canonical ensemble, that is, for fixed values of temperature and chemical potentials ( $\mu_{1}$ for silver atoms and $\mu_{2}$ for anions) the number of particles of both species can fluctuate. In the present work, we have considered the temperature fixed as $T=300 \mathrm{~K}$ and the chemical potential for anions arbitrarily fixed as $\mu_{2}=0$ in most of the cases and $\mu_{2}=-0.327 \mathrm{eV}$ (a more realistic value) in some cases in order to compare the effect of this chemical potential.

We have calculated adsorption isotherms, that is, coverage degree $\left(\theta_{\mathrm{Ag}}\right.$ for the fraction of sites of the first monolayer occupied by silver atoms and $\theta_{\text {anion }}$ for the fraction of sites of the second monolayer occupied by anions) as a function of the chemical potential $\mu_{1}$.

In most cases, we have considered as initial state for each chemical potential, the final state of the previous chemical potential, running from more negative through more positive values of $\mu_{1}$. The chemical potential was increased in $0.01 \mathrm{eV}$ steps in regions where $\theta_{\mathrm{Ag}}$ changes appreciably and in $0.05 \mathrm{eV}$ steps otherwise.

The simulation consists in performing (for each value of $\mu_{1}$ ) a certain number of Monte Carlo steps (MCS) in order to equilibrate the system and then another set of MCS in order to obtain the average values of $\theta_{\mathrm{Ag}}$ and $\theta_{\text {anion. }}$. Each MCS implies the realization of $2 \times M$ trials. Each trial consists on the random selection of one of the two monolayers, with equal probability, and then, the random selection of one lattice site.

If the first monolayer was selected, there are two possibilities.

(a) If the site is empty (occupation 0), the creation of an adsorbate atom is attempted (yielding occupation 1). In this case we set $\Delta N_{\mathrm{Ag}}=1$ and $\Delta N_{\text {anion }}=0$, were $N_{\mathrm{Ag}}$ is the number of silver atoms and $N_{\text {anion }}$ is the number of anions present.

(b) If the site is occupied by an adsorbate atom (occupation 1 ), its desorption is attempted (occupation 0). In this case we set $\Delta N_{\mathrm{Ag}}=-1$ and $\Delta N_{\text {anion }}=0$.

If the second monolayer was selected, there are two possibilities.

(a) If the site is empty (occupation 0), the creation of an anion is attempted (yielding occupation 2). In this case, we have $\Delta N_{\mathrm{Ag}}=0$ and $\Delta N_{\text {anion }}=1$.

(b) If the site is occupied by an anion (occupation 2), its elimination is attempted (occupation 0 ). In this case we have $\Delta N_{\mathrm{Ag}}=0$ and $\Delta N_{\text {anion }}=-1$.

In all the cases, the change is accepted with probability

$$
P=\min \left\{1, \exp \left[-\frac{\Delta E-\mu_{1} \Delta N_{\mathrm{Ag}}-\mu_{2} \Delta N_{\text {anion }}}{k_{B} T}\right]\right\},
$$

where $\Delta E$ represents the difference between the energies of the final and initial states and $k_{B}$ is the Boltzmann constant.

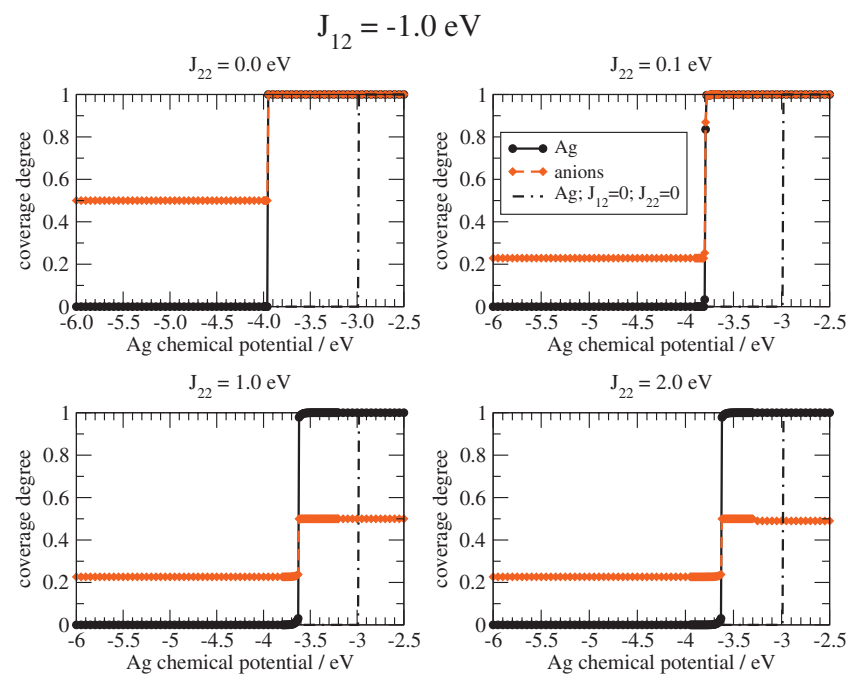

FIG. 1. Adsorption isotherms for $J_{12}=-1.0 \mathrm{eV}$ and four values of $J_{22}$, as indicated. Each graphic contains the coverage degree of adatoms $\theta_{\mathrm{Ag}}$ (full lines, closed circles) and the coverage degree of anions $\theta_{\text {anion }}$ (dashed lines, full diamonds) for each case as a function of the chemical potential of silver. The chemical potential for anions is considered as $\mu_{\text {anion }}=0.0 \mathrm{eV}$. The point-broken line denotes $\theta_{\mathrm{Ag}}$ for the case $J_{12}=J_{22}=0.0 \mathrm{eV}$. For each chemical potential the initial state corresponds to the final state of the previous chemical potential.

\section{RESULTS AND DISCUSSION}

The simulations were performed considering two adjacent square lattices of size $100 \times 100$. Adsorption isotherms were calculated as the mean value of $\theta_{\mathrm{Ag}}$ and $\theta_{\text {anion }}$ as a function of the chemical potential of $\mathrm{Ag}\left(\mu_{1}\right)$. For each value of $\mu_{1}$, we have performed $M_{1}$ MCS in order to equilibrate the system and $M_{2} \mathrm{MCS}$ for the calculation of the average values. The values of $M_{1}$ and $M_{2}$ were $10^{6}$ for the zones of interest (near the jumps of the isotherms) and $10^{5}$ for the rest of the isotherm.

Figure 1 shows four adsorption isotherms for $J_{12}$ $=-1.0 \mathrm{eV}$ and $\mu_{2}=0 \mathrm{eV}$. We illustrate there the cases $J_{22}$ $=0.0,0.1,1.0$, and $2.0 \mathrm{eV}$. Together with the isotherms of $\theta_{\mathrm{Ag}}$ and $\theta_{\text {anion }}$, we show also the isotherm $\left(\theta_{\mathrm{Ag}}\right)$ for the case $J_{12}$ $=J_{22}=0.0 \mathrm{eV}$ (which is equivalent to the absence of anions), in order to illustrate the effect of the anions. We can see that due to the influence of the anions, the jump in $\theta_{\mathrm{Ag}}$ is shifted toward more negative chemical potentials. In all these cases, the transition between $\theta_{\mathrm{Ag}}=0$ and $\theta_{\mathrm{Ag}}=1$ remains abrupt.

For $J_{22}=0 \mathrm{eV}$, that is, for negligible interactions between anions, $\theta_{\text {anion }}$ changes from 0.5 (when $\theta_{\mathrm{Ag}}=0$ ) to 1 (when $\theta_{\mathrm{Ag}}=1$ ). In the region previous to $\mathrm{Ag}$ adsorption, $\theta_{\text {anion }}=0.5$ because $\mu_{2}=0.0 \mathrm{eV}$. In fact, $\theta_{\text {anion }}=0.5$, corresponds to the value expected for a Langmuir isotherm (without lateral interactions between anions) with $E_{\mathrm{ads}}=0 \mathrm{eV}$ (absence of silver atoms). Under these conditions, the anions are distributed at random.

When $J_{22}>0 \mathrm{eV}$, in the absence of silver atoms, we have a coverage degree of $\theta_{\text {anion }} \approx 0.22$. This coverage degree corresponds to the equilibrium value for $\mu_{2}=0.0 \mathrm{eV}$, adsorption energy 0 (for the anions, in the absence of silver atoms) and repulsive interactions between anions (see Ref. 28). In fact, in this reference the authors have studied the adsorption isotherms for different repulsive interactions between ad- 

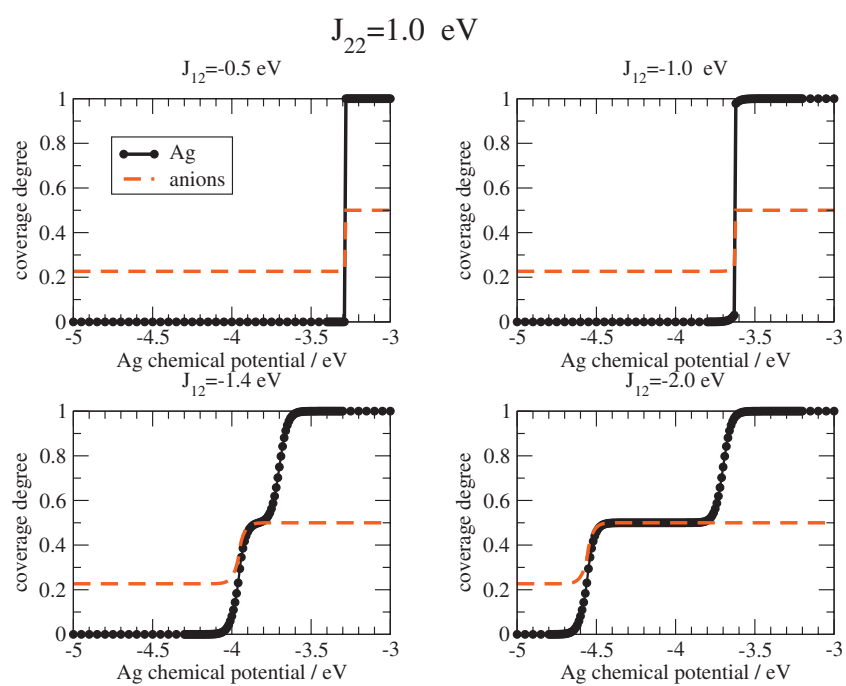

FIG. 2. Adsorption isotherms for $J_{22}=1.0 \mathrm{eV}$ and four values of $J_{12}$, as indicated. Each graphic contains the coverage degree of adatoms $\theta_{\mathrm{Ag}}$ (full lines, closed circles) and the coverage degree of anions $\theta_{\text {anion }}$ (dashed lines, no symbol) for each case as a function of the chemical potential of silver. The chemical potential for anions is considered as $\mu_{\text {anion }}=0 \mathrm{eV}$. For each chemical potential, the initial state correspond to the final state of the previous chemical potential.

sorbed particles, and found the asymptotic value of $\theta_{\text {anion }}$ $\approx 0.226$ at $\mu_{2}=0.0 \mathrm{eV}$ for infinite repulsion between adsorbates. The same value can also be obtained via analytical calculations in the framework of the quasichemical approximation. ${ }^{19}$ In this approach, which provides excellent results in the range of low concentrations $(0 \leq \theta<1 / 4)$, the analytical expression of the adsorption isotherm for a square lattice gas of interacting particles can be written as ${ }^{19}$

$$
e^{(\mu-2 w) / k_{B} T}=\left[\frac{(b-1+2 \theta)}{(b+1-2 \theta)}\right]^{2} \frac{1-\theta}{\theta},
$$

where $b=\sqrt{1-4 \theta(1-\theta)\left(1-e^{-w / k_{B} T}\right)}$ and $w$ is the nearestneighbor interaction energy that corresponds to repulsive (attractive) interaction for $w>0(w<0)$. Then, solving Eq. (4) for $\mu=0$ and $w \rightarrow \infty$, we obtain $\theta \approx 0.22$. In our case the repulsions are not infinite but already important enough to be close to the limiting value.

When $\theta_{\mathrm{Ag}}=1$, we find $\theta_{\text {anion }}=1$ for low values of $J_{22}$ and $\theta_{\text {anion }}=0.5$ for high values of $J_{22}$. This situation corresponds to chessboardlike structures, typical of adsorption isotherms with repulsive interactions. The isotherms for $J_{22}=1$ and 2 $\mathrm{eV}$ present a jump in $\theta_{\mathrm{Ag}}$ at a more positive $\mu_{1}$ value than the cases with small $J_{22}$. The general trend will be illustrated later. It must also be noticed that in all the cases corresponding to $J_{12}=-1.0 \mathrm{eV}$, there is an abrupt jump between $\theta_{\mathrm{Ag}}$ $=0$ and $\theta_{\mathrm{Ag}}=1$.

In order to analyze the effect of the chemical potential of anions, some simulations have been made with $\mu_{2}$ $=-0.327 \mathrm{eV}$ (not shown here). We have found that the qualitative behavior is very similar to the case with $\mu_{2}=0.0 \mathrm{eV}$, except that, for the areas with $\theta_{\mathrm{Ag}}=0, \theta_{\text {anion }}$ is also equal to 0 .

Figure 2 shows four adsorption isotherms for $J_{22}$ $=1.0 \mathrm{eV}$, for the case of $\mu_{2}=0 \mathrm{eV}$. We illustrate the cases $J_{12}=-0.5,-1.0,-1.4$, and $-2.0 \mathrm{eV}$. We can see that as $J_{12}$ increases, the chemical potential for the sudden increase in

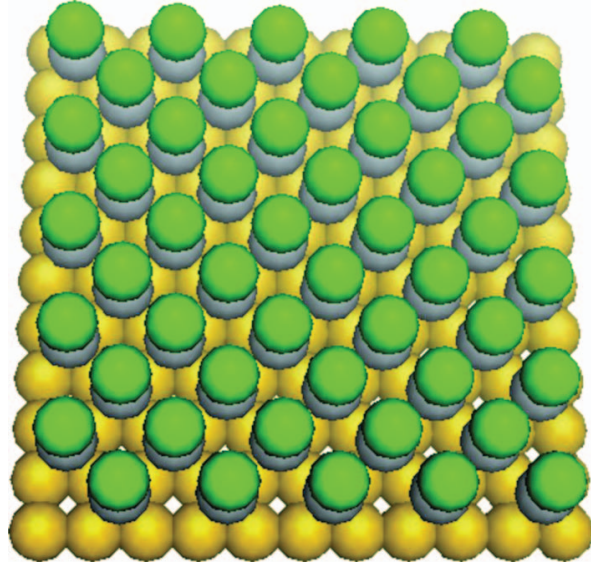

FIG. 3. Illustration of a portion of the system for the situation corresponding to $\theta_{\mathrm{Ag}}=\theta_{\text {anion }}=0.5$. Such structures are found in the cases with $J_{22}=1.0 \mathrm{eV}$ and high values of $\left|J_{12}\right|$. Light gray spheres in the underlying lattice: gold atoms. Gray spheres on hollow sites: silver atoms. Dark gray spheres: anions.

$\theta_{\text {Ag }}$ that we shall denote with $\mu_{\text {coex }}$, shifts toward more negative values. This is an indication that the presence of anions favors the adsorption of silver.

For low values of $J_{12}$, we find an abrupt jump between $\theta_{\mathrm{Ag}}=0$ and $\theta_{\mathrm{Ag}}=1$, as expected for first order phase transitions. However, for higher values of $J_{12}$, we find that the jump becomes smoother and we have the appearance of an intermediate region, with $\theta_{\mathrm{Ag}}=\theta_{\text {anion }}=0.5$. This region corresponds to the situation illustrated in Fig. 3 for a $10 \times 10$ surface. We can see that the chessboard configuration of the anions, due to the repulsive lateral interactions between them, induces the same structure for the underlying monolayer of silver atoms. These results show that this simple model can explain the appearance of expanded structures, as observed experimentally for several UPD systems.

The regions with $\theta_{\mathrm{Ag}}=1$ and $\theta_{\text {anion }}=0.5$ presents the structure shown in Fig. 4 for a $10 \times 10$ surface. In these regions, the silver monolayer is complete and the anions are arranged in a chessboardlike structure. In the following, we consider the dependence of $\mu_{\text {coex }}$ with $J_{12}$ and $J_{22}$.

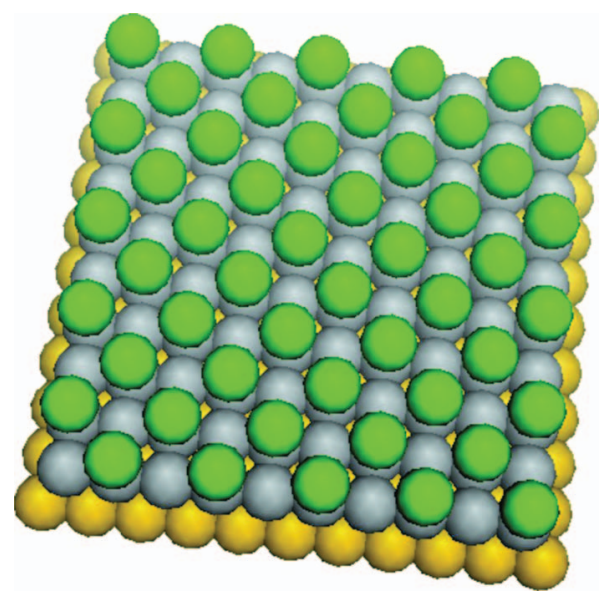

FIG. 4. Illustration of a portion of the system for the situation corresponding to $\theta_{\mathrm{Ag}}=1$ and $\theta_{\text {anion }}=0.5$. Such structures are found in the cases with $J_{22}$ $=1.0 \mathrm{eV}$. Light gray spheres in the underlying lattice: gold atoms. Gray spheres on hollow sites: silver atoms. Dark gray spheres: anions. 


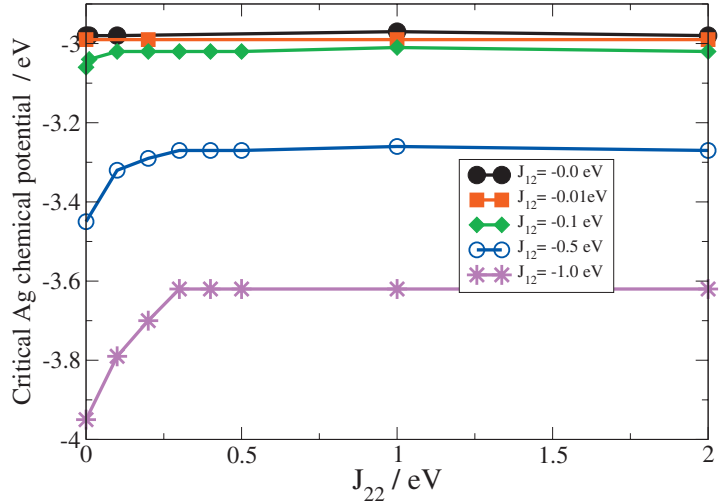

FIG. 5. Plots of the threshold chemical potential $\mu_{\text {coex }}$ for the appearance of $\mathrm{Ag}$ adatoms on the surface, as a function of $J_{22}$ for several values of $J_{12}$, as indicated in the figure. The chemical potential for anions is considered as $\mu_{\text {anion }}=0.0 \mathrm{eV}$.

Figure 5 shows the variation of $\mu_{\text {coex }}$ with $J_{22}$ for several values of $J_{12}$. We can see that for low absolute values of the attractive interaction $J_{12}, \mu_{\text {coex }}$ is almost independent of $J_{22}$ and similar to the value found for Ag adsorption in the absence of anions. On the other hand, for high values of $J_{12}$, we see that $\mu_{\text {coex }}$ increases initially with $J_{22}$, but approximately from $J_{22}=0.5 \mathrm{eV}$ on, it remains almost constant. That means that for large enough anion-anion interactions, we can consider that the isotherms do not depend on that parameter. However, for weak anion-anion interactions, the value of $\mu_{\text {coex }}$ becomes more negative, indicating that the adsorption of the silver monolayer is more favorable.

Figure 6 shows the variation of $\mu_{\text {coex }}$ with $-J_{12}$ for several values of $J_{22}$. We can see that as $\left|J_{12}\right|$ increases, $\mu_{\text {coex }}$ becomes more negative. This fact shows that stronger interactions between silver atoms and anions favor the monolayer adsorption and the observation of UPD. The $\mu_{\text {coex }}-J_{22}$ correlation becomes almost linear for $J_{22} \rightarrow 0$, so that under these conditions the shift in the UPD peak could be used as a direct measure of the adatom-ion interactions.

Figure 7 shows adsorption isotherms (coverage degree of silver atoms as a function of the chemical potential) for the case of $J_{22}=0.4 \mathrm{eV}$ and several values of $J_{12}$. The behavior of the isotherms is the same for all values of $J_{22}$ $\geq 0.4 \mathrm{eV}$. In these cases, for $\left|J_{12}\right| \geq 1.3 \mathrm{eV}$, it can be ob-

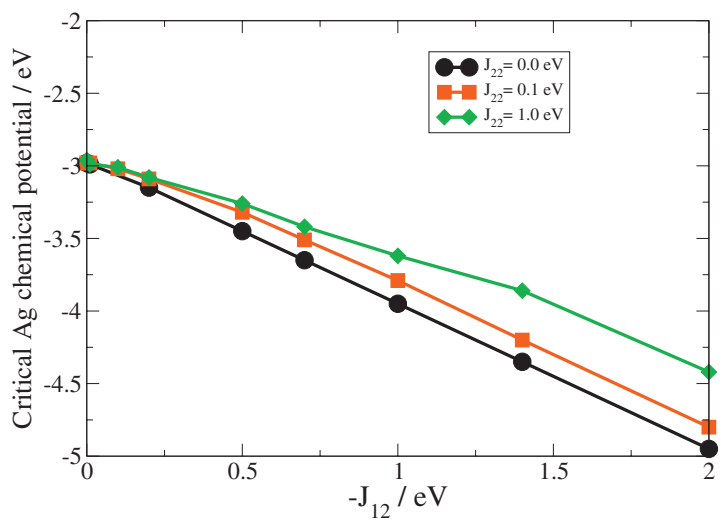

FIG. 6. Plots of the threshold chemical potential $\mu_{\text {coex }}$ as a function of $J_{12}$ for several values of $J_{22}$, as indicated in the figure. The chemical potential for anions is considered as $\mu_{\text {anion }}=0.0 \mathrm{eV}$.

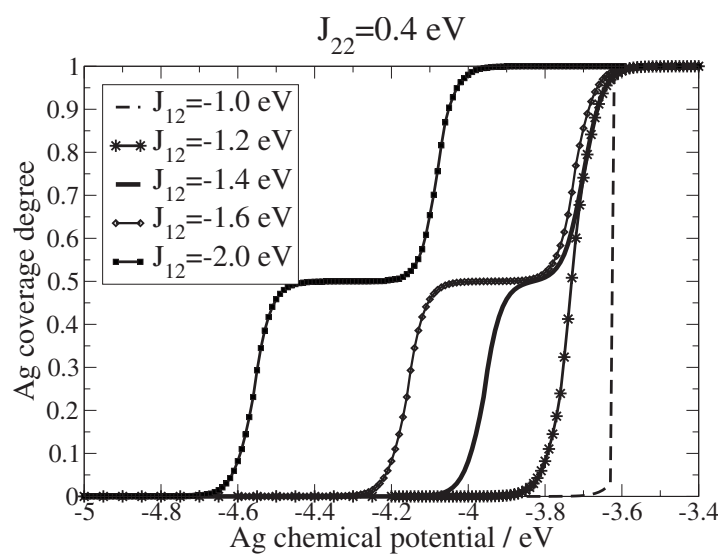

FIG. 7. Adsorption isotherms for $J_{22}=0.4 \mathrm{eV}$ and several values of $J_{12}$, as indicated. The coverage degree of the $\mathrm{Ag}$ atoms, $\theta_{\mathrm{Ag}}$, is plotted as a function of their chemical potential. The chemical potential for anions is considered as $\mu_{\text {anion }}=0.0 \mathrm{eV}$. For each chemical potential the initial state corresponds to the final state of the previous chemical potential.

served the appearance of a plateau at $\theta_{\mathrm{Ag}}=0.5$. It corresponds to the formation of a expanded structure as shown in Fig. 4. Thus, we can define the values of $J_{22}$ and $J_{12}$ for which an expanded structure is expected. In the zone of $J_{22}<0.4 \mathrm{eV}$ we found a more complex behavior due to the similarities in the values of $J_{22}$ and $J_{11}\left(\left|J_{11}\right|=0.28 \mathrm{eV}\right)$. This region will be analyzed in detail in a future work.

\section{CONCLUSIONS}

In the present work, we have proposed a general simulation scheme in terms of a lattice model to consider the change of the electrochemical stability of adatoms upon interaction with anionic species that may coadsorb from the solution. The model aims to describe the situation where the anions are adsorbed on top of the metal atoms when being deposited. The physical picture underlying this assumption is that a partial charge transfer from the adatom to the surface may enhance anion adsorption. Simulations of adsorption isotherms were performed for a system where the metalmetal interactions should represent the $\mathrm{Ag} / \mathrm{Au}(100)$ system, considering different metal-anion and anion-anion interactions. As the interaction between adatoms and anions increases, the monolayer adsorbs at more negative chemical potentials. The extent of this shift correspond to a few hundreds of millivolts in the electrochemical scale, thus delivering the correct order of magnitude for this effect. When the interactions between the adatoms and the anions is relatively large, expanded structures are found, in qualitative agreement with experimental results.

In order to come to a closer agreement with experimental results, we can incorporate several modifications to the present model in the future. For instance, it is desirable to extend the present model to consider non-nearest neighbor interactions, which may be especially important in the case of the electrostatic anion-anion interactions. Adsorption of anions on the substrate surface can also be considered. 


\section{ACKNOWLEDGMENTS}

The authors thank CONICET (Argentina), Universidad Nacional de San Luis, Project No. 322000, National Agency of Scientific and Technological Promotion (Argentina) under Project Nos. 33328 PICT 2005 and 946 PICT 2006, and CONICET (Argentina) though Project PIP No. 112-20080101332.

${ }^{1}$ D. M. Kolb, M. Przasnyski, and H. Gerischer, J. Electroanal. Chem. 54, 25 (1974).

${ }^{2}$ S. Trasatti, Z. Phys. Chem., Neue Folge 98, 75 (1975).

${ }^{3}$ E. Budevski, G. Staikov, and W. J. Lorenz, Electrochemical Phase Formation and Growth (VCH, Weinheim, 1996).

${ }^{4}$ E. Budevski, G. Staikov, and W. J. Lorenz, Electrochim. Acta 45, 2559 (2000).

${ }^{5}$ E. P. M. Leiva, Current Topics in Electrochemistry (Council of Scientific Information, Trivandrum, India, 1993), Vol. 2, p. 269.

${ }^{6}$ E. P. M. Leiva, Electrochim. Acta 41, 2185 (1996).

${ }^{7}$ W. Plieth, W. J. Lorenz, and G. Staikov, J. Solid State Electrochem. 8, 941 (2004).

${ }^{8}$ M. C. Giménez, M. G. Del Pópolo, and E. P. M. Leiva, Electrochim. Acta 45, 699 (1999).

${ }^{9}$ M. C. Giménez and E. P. M. Leiva, Langmuir 19, 10538 (2003).

${ }^{10}$ C. Chen, S. M. Vesecky, and A. J. Gewirth, J. Am. Chem. Soc. 114, 451 (1992).

${ }^{11}$ K. Ogaki and K. Itaya, Electrochim. Acta 40, 1249 (1995).

${ }^{12}$ S. Garcia, D. Salinas, C. Mayer, E. Schmidt, G. Staikov, and W. Lorenz, Electrochim. Acta 43, 3007 (1998)
${ }^{13}$ M. J. Esplandiu, M. A. Schneeweiss, and M. A. Kolb, Phys. Chem. Chem. Phys. 1, 4847 (1999).

${ }^{14}$ E. Herrero, L. J. Buller, and H. D. Abruña, Chem. Rev. (Washington, D.C.) 101, 1897 (2001).

${ }^{15}$ C. G. Sánchez, S. A. Dassie, and E. P. M. Leiva, Langmuir 18, 6628 (2002).

${ }^{16}$ C. Sánchez and E. P. M. Leiva, Electrochim. Acta 45, 691 (1999).

${ }^{17}$ P. A. Rikvold, J. Zhang, Y. E. Sung, and A. Wieckowski, Electrochim. Acta 41, 2175 (1996).

${ }^{18}$ L. Blum, D. A. Huckaby, and M. Legault, Electrochim. Acta 41, 2207 (1996).

${ }^{19}$ T. L. Hill, An Introduction to Statistical Thermodynamics (Dover, New York, 1986).

${ }^{20}$ D. Nicholson and N. G. Parsonage, Computer Simulation and Statistical Mechanics of Adsorption (Academic, London, 1982).

${ }^{21}$ S. G. García, D. Salinas, C. Mayer, J. R. Vilche, H.-J. Pauling, S. Vinzelberg, G. Staikov, and W. J. Lorenz, Surf. Sci. 316, 143 (1994).

${ }^{22}$ N. Ikemiya, K. Yamada, and S. Hara, Surf. Sci. 348, 253 (1996).

${ }^{23}$ M. C. Giménez, M. G. Del Pópolo, E. P. M. Leiva, S. G. García, D. R. Salinas, C. E. Mayer, and W. J. Lorenz, J. Electrochem. Soc. 149, E109 (2002).

${ }^{24}$ M. C. Giménez, M. G. Del Pópolo, and E. P. M. Leiva, Langmuir 18, 9087 (2002).

${ }^{25}$ Z. Shi, S. Wu, and J. Lipkowski, Electrochim. Acta 40, 9 (1995).

${ }^{26}$ M. C. Giménez, A. J. Ramirez-Pastor, and E. P. M. Leiva, Surf. Sci. 600, 4741 (2006).

${ }^{27}$ Z. Shi, S. Wu, and J. Lipkowski, Electrochim. Acta 40, 15 (1995).

${ }^{28}$ F. Bulnes, A. J. Ramirez-Pastor, and G. Zgrablich, Adsorpt. Sci. Technol. 23, 81 (2005). 